\title{
Lupus Nephritis
}

An International Meeting held at University College and Middlesex School of Medicine, Thursday October 20 1988.

Programme organisers and co-chairmen: David Isenberg, Guy Neild and Michael Snaith.

\section{Lupus nephritis: a review}

\author{
V.B. Dhillon, M.L. Snaith and D.A. Isenberg \\ Bloomsbury, Rheumatology Unit, Arthur Stanley House, Tottenham Street, London WIP 9PG, UK.
}

\section{Introduction}

An international meeting on lupus nephritis was held in October 1988 at the Middlesex Hospital, London (under the auspices of the University College and the Middlesex School of Medicine). The audience consisted of rheumatologists and nephrologists with an interest in lupus nephritis. The meeting served as a forum for discussion about a number of important issues; it was not anticipated that a major consensus view on the questions being raised would necessarily emerge.

Renal involvement complicating systemic lupus erythematosus (SLE) is potentially life-threatening. Serious lupus nephritis was considered rare until the 1940s. ${ }^{1}$ Recent prevalence figures for lupus nephritis have been quoted as $71 \%$ of black and $51 \%$ of white patients with SLE. ${ }^{2}$ There is, however, obvious selection bias within any group. In the Bloomsbury Rheumatology Unit, for example, only $30 \%$ of the first 100 patients followed up for 6 months to 11 years had renal involvement as defined by combinations of the following criteria ${ }^{3}$

(i) diastolic blood pressure $>90 \mathrm{mmHg}$

(ii) oedema requiring diuretic therapy

(iii) systolic hypertension

(iv) proteinuria $>0.5 \mathrm{~g} / 24$ hours

(v) creatinine clearance $<60 \mathrm{ml} /$ minute

(vi) serum creatinine $>124 \mu \mathrm{mol} / \mathrm{l}$

\section{Diagnosis}

Lupus nephritis may present with hypertension, oedema, weight gain or oliguria, or it may be asymptomatic. Dipstick testing is a rapid though perhaps over-sensitive method of showing haematuria and/or proteinuria. Light microscopy of fresh urine may show red or white cell casts.
Laboratory indicators include raised serum urea and creatinine, hypoalbuminaemia, raised dsDNA.

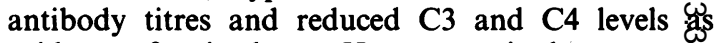
evidence of active lupus. However, raised serum urga and creatinine might also indicate long term damage rather than current active disease. Glomerular filtra tion rate (GFR), preferably measured by isotope clearance, is reduced. Twenty four-hour urina creatinine clearance, which relies upon complete and accurately timed collection of urine, is also rede्gced and protein excretion is increased.

Definitive diagnosis is made by percutaneous biopsy, and Dr Gwyn Williams (Abstract $p=3 \frac{4}{3} 3$ recommended that renal biopsy should be performegd in all lupus patients with persistent haematuria and/är proteinuria, and in all patients with reduced GFR

However, the prevalence of clinically silent luptss nephritis is between 30 and $50 \%$. In such cases deposits and proliferative glomerular changes hape been demonstrated in biopsies from patients with $\stackrel{\overrightarrow{0}}{\mathrm{~F}}$ clinical evidence of renal disease. ${ }^{4,5}$ The role of biopsy and therapy in this context remains uncertain.

\section{Histology}

Light microscopy provides information about the classification of types of nephritis, tubular damage, interstitial disease, activity and chronicity indice. Immunofluorescence indicates the composition and type of immune deposits, and electron microscopy (EM) demonstrates the site of deposits.

Professor D.R. Turner (Abstract p.342) outlined the histological classification of lupus nephritis. Acute or active damage is indicated by: (i) disruption of capilary walls, (ii) polymorphs and karyorrhexis, (i haematoxophil bodies (the tissue equivalent of IEE cells, which when present are pathognomonic of lupus nephritis), (iv) cellular crescents, (v) wire loops, (ด्)

(C) The Fellowship of Postgraduate Medicine, 19\%9 
hyaline thrombi, (vii) fibrin thrombi and (viii) segmental fibrin deposits.

Histological abnormality may also reflect damage to the kidney in the past, in the form of chronic, irreversible sclerotic lesions. Renal biopsy specimens thus provide much valuable information regarding the diagnosis, prognosis, and the need for initiation, continuation or cessation of treatment.

The WHO classification of lupus nephritis is shown in Table I. Grades III and IV are the most severe forms.

Table I WHO classification of lupus nephritis

\begin{tabular}{ll}
\hline I & Normal by light microscopy \\
II & Pure mesangial changes \\
III & Focal and segmental glomerulonephritis \\
IV & Diffuse proliferative glomerulonephritis \\
V & Diffuse membranous glomerulonephritis \\
VI & Advanced sclerosing glomerulonephritis \\
\hline
\end{tabular}

\section{Value of biopsy-a brief historical review}

When discussing the value of a particular investigation, the concept of 'marginal benefit' is used. This can be defined as the difference between what is known before and after that investigation. Fries et al. ${ }^{6}$ found that renal biopsy contains important prognostic information but that this is less than that of even the simplest clinical classifications. They felt that renal biopsy needed reassessment for marginal benefit rather than for absolute predictive ability. WhitingO'Keefe et al. ${ }^{7}$ applied stepwise linear regression analysis to data collected over a 12 month period after renal biopsy in 130 SLE patients, to see if biopsy added any useful information to the clinical data. They found that histological classification did not add significantly to the predictive power of the 'before biopsy' model, but that certain features, percentage glomerular sclerosis and subendothelial deposits on EM, increased the ability to predict the effect of 12 months of treatment of active lupus nephritis. Another paper from the same group ${ }^{8}$ found that renal biopsy did not add important prognostic information over and above the history, examination and laboratory tests.

The presence of subendothelial deposits appears to be of value: Dillard et al. ${ }^{9}$ studied 20 renal biopsies from 13 patients with SLE. They found that, although subendothelial deposits were not invariably associated with severe renal disease, when the latter existed large subendothelial deposits were found. They tended to disappear with treatment and clinical improvement. Tateno et al. ${ }^{10}$ found that the degree of proteinuria and histological activity correlated with the amount of subendothelial deposits. C3 level and creatinine clearance correlated negatively with these deposits.

\section{Problems with biopsy}

Histological classification can be problematic, in that SLE produces a wide range of abnormalities affecting the glomeruli, tubulo-interstitial compartment, and occasionally blood vessels. Inter-observer and interglomerular variation are important, and a biopsy is, by definition, a single observation in a process which will vary with time. These isolated observations often do not take into account exacerbations in disease, and movement between different WHO grades of disease can occur. They may in fact be as frequent as $\mathbf{4 0}$ to $50 \%$ over 7 to 10 years.

\section{Pathogenesis (recent experimental work)}

\section{Anti-DNA antibodies}

Dr Dan Eilat (Abstract p.341) discussed the role of anti-DNA antibodies including the paradox that DNA itself is a rather poor immunogen. This raises the possibility that something other than the deposition of DNA - anti-DNA immune complexes might be responsible for the immunopathology of renal lupus. Eilat presented results of in vitro studies using monoclonal anti-DNA antibodies produced by the hybridoma technique from non-immunized diseased NZB/ NZW mice. He confirmed the basic structural requirements for cross-reactive antigens by establishing the primary sequence, and building a threedimensional computer model of the antibody. In vivo studies have been performed using the isolated perfused rat kidney, and the IgG anti-DNA monoclonal antibody A52 was shown to have a particular affinity for renal tissue. His work supports a newer pathogentic model: that anti-DNA antibodies react with non-DNA, cross-reactive renal antigens.

\section{Animal models}

Dr P.J. Hoedemaeker (Abstract p. 341) pointed out the problem of inducing auto-immune nephritis in animal models using complete Freund's adjuvant. Down-regulation of autoimmunity occurs as a result of development of antigen-specific suppressor $T$ cells, such that ultimately auto-immunity disappears. Graftversus-host $(\mathrm{GvH})$ disease provides a good model of lasting auto-immune nephritis by prolonged B cell stimulation as a result of lasting chimerism of lymphocytes. Experimentally produced $\mathrm{GvH}$ disease and lupus nephritis are similar with respect to morphology, functional changes, antibodies and severity of disease produced.

Dr Alfred D. Steinberg (Abstract p. 342) has studied murine lupus extensively, and a model for pathogenesis has been developed: he discussed the 
concept of polyclonal B cell stimulation by various endogenous and/or exogenous factors. A vicious circle of antigen dependent $B$ cell expansion ensues, with the production of pathogenetic auto-antibodies and thus disease.

The mechanism of photosensitivity has been suggested by studies in BXSB mice: ultraviolet B light produces polyclonal activation via interleukin-I, as well as affecting the handling of antigen-antibody complexes and antigen presentation.

He raised the interesting question of the role of activation of highly conserved oncogene sequences in disease production.

\section{Complement}

Dr M. Walport (Hammersmith Hospital, London) presented work on the role of complement in lupus nephritis. C5a is a chemoattractant for neutrophils, $\mathrm{C} 3 \mathrm{~b}$ ligates leucocyte receptors. The membrane attack complex has been extensively studied and shown to disrupt the cell membrane, to effect an increase in prostaglandin $\mathrm{E}$ and thromboxane $\mathrm{A} 2$ production by glomerular epithelium, and to stimulate interleukin-I and oxygen radical release. Loss of complement receptor 1 appears to be associated mainly with subepithelial deposition of IgG and C3.

\section{Vascular injury}

Dr G.H. Neild (Abstract p. 341) outlined four types of vascular injury: arterial, arteriolar, glomerular and endothelial. While the mechanism of endothelial injury is unknown, humoral factors such as antiendothelial cell antibodies, cell mediated immunity with production of cytokines, platelets and platelet activating factor may all play a role. The role of anti-cardiolipin antibodies in relation to the formation of glomerular microthrombi and progressive glomerular sclerosis is still uncertain. The role of antibodies was discussed with particular emphasis on anti-endothelial cell antibodies which have been demonstrated by ELISA in up to $74 \%$ of SLE patients. ${ }^{11}$

\section{Treatment}

Professor J. Stewart Cameron (Abstract p. 343) said that satisfactory trials have been few and difficult to perform because of the variable presentation and course of SLE, and therefore must be analysed with caution. Survival of WHO grades III and IV has improved over the past 30 years from a two year survival of less than $10 \%$ to a ten year survival of $87 \%$ in the best series.

There are two phases in the treatment of lupus nephritis: (i) the active phase, where it is important to suppress disease, and (ii) the chronic phase, whet minimization of treatment side effects is crucial.

It is generally accepted that aggressive treatment needed in cases of severe histological involvement, and in renal failure or nephritic syndrome. In these cases, combined steroid and cytotoxic treatment is bette than steroids alone, and a number of studies suppost this. Austin et al. ${ }^{12}$ showed that, compared with hig dose oral prednisolone alone, intravenous cyclophosphamide reduces the risk of end-stage renal failune with few serious complications. Balow et al. ${ }^{13}$ showec that cytotoxic treatment reduces the likelihood of progressive renal scarring in lupus nephritis. Carette $\vec{\theta}$ al. ${ }^{14}$ found that single drug oral cytotoxic therapy and prednisolone was most beneficial in lupus patiens with an intermediate chronicity index.

The route of administration of these drugs remaing controversial. Pulsed cyclophosphamide, pulsed methylprednisolone and low dose oral steroids from the outset appear to reduce the risk of steroid toxicity However, Kimberley ${ }^{15}$ reported that inhibition of prostaglandin synthesis by high dose steroids mas compromise renal function in lupus patients, as $\dot{q}$ appears that maintenance of renal haemodynamics in lupus patients may depend on enhanced renal prostage landin production.

Dr Neild stressed the difficulty of recognizing haemolytic uraemic syndrome, as indicated by mice roangiopathic haemolytic anaemia, and the appropriate management of this rare but important comp tion of lupus nephritis.

Professor Cameron mentioned the controversi बa् role of plasma exchange, and the use of cyclosporin as a steroid sparing agent, but this latter drug often has no effect in severe active disease. In the future $\vec{\theta}$ rational approach to treatment might lie in the production of human-mouse hybrid monoclona antibodies directed against various $T$-cell antigens. Renal transplantation in lupus nephritis is weth tolerated, and lupus nephritis only rarely recurs grafted kidneys. ${ }^{16}$

\section{Monitoring treatment}

Laboratory variables Cameron et al. ${ }^{17}$ found comple ment levels and dsDNA binding useful in the diagnosis of SLE, but not so useful as a measure of active. nephritis. Pillemer et al. ${ }^{18}$ found that abnormal $\mathrm{CB}$ predicts the degree of persistent active glomerulonephritis, but duration of abnormal C3 or raised antio DNA antibody titres are less consistent predictors acquisition of chronic irreversible renal lesions. recent study has shown that measuring the breakdown fragment C4d on its own or as a ratio with $\mathrm{C} 4$ miglef also be a useful guide to disease activity. ${ }^{19}$

Biopsy Stamenkovic et al. ${ }^{20}$ gave treatment based of 
renal histology to 56 SLE patients. The mean followup period from first biopsy was 8.2 years, $5.3 \%$ were dialysis dependant and $94.7 \%$ resumed normal renal function. They found that biopsy revealed valuable data regarding renal status irrespective of clinically apparent renal disease and the authors felt that treatment based on biopsy alone could considerably improve renal survival in SLE. Similarly Balow ${ }^{21}$ reports that renal histology may identify the efficacy of various therapeutic agents sooner than renal function changes as measures of outcome.

\section{Prognosis}

Cheigh et al. ${ }^{22}$ reported that survival rates with end-stage renal failure following lupus nephritis were comparable with those of the general dialysis population: actuarial survival rate one year after dialysis was $91 \%$, two years $79 \%$ and five years after dialysis $69 \%$. Patients on long term dialysis with SLE tended to remain clinically inactive despite persistently abnormal serology. Ziff and Helderman ${ }^{16}$ also report that patients with SLE in advanced renal failure have remission of non-renal features but they report an improvement in serological abnormalities. Coplon et $a l .,^{23}$ in their 12 year follow-up of 28 patients with lupus nephritis, found that long term clinical course was comparable to that of patients with end-stage renal failure associated with other disorders.

Age and a chronicity index have been shown by Austin et al. ${ }^{24}$ to be highly significant prognosticators. Histology is useful, in that end-stage histology represents end-stage renal failure. Diffuse proliferative glomerulonephritis (WHO IV) carries a worse prognosis if untreated, and subendothelial deposits indicate poorer prognosis because of the tendency to develop into proliferative disease.

Correia et al ${ }^{25}$ performed a retrospective analysis of 138 patients with SLE. Forty two patients died over a

\section{References}

1. Meuhrcke, R.C., Kark, R.M., Pirani, C.L. \& Pollak, V.E. Lupus nephritis: a clinical and pathologic study based on renal biopsies. Medicine (Baltimore) 1957, 35: $1-145$.

2. Studenski, F., Allen, N.B., Caldwell, D.S., Rice, J.R. \& Polisson, R.P. Survival in systemic lupus erythematosus: a multivariate analysis of demographic factors. Arth Rheum 1987, 30: 1326-1332.

3. Morrow, W.J.W., Isenberg, D.A., Todd-Pokropek, A., Parry, H.F. \& Snaith, M.L. Useful laboratory measurements in the management of systemic lupus erythematosus. $Q J$ Med 1982, 202: 125-138. period of 20 years and data were analysed from 41 . In most cases the cause of death was multiple; 27 developed terminal renal failure, and 13 of these died a few weeks or months after starting dialysis, the main causes being active lupus or infection. An important cause of death was atherosclerosis and five out of the seven deaths due to this were of women under 40 years of age. Leung and Boulton-Jones ${ }^{26}$ replied to this paper, reporting a series of seven deaths in patients with lupus nephritis over a period of 10 years, five of whom died as a result of severe acute pulmonary disease (one pulmonary embolism, one diffuse intraalveolar haemorrhage and two in whom the predominant histology was pulmonary oedema). They were all women, with a mean age of 31 years.

\section{Conclusion}

It is clear that much progress has been made in our understanding of lupus nephritis, both at an experimental and at a clinical level. However, more studies are required, particularly with reference to drug treatment. There is a need for multi-centre trials with follow-up for at least 10 years. The problem of clinically silent lupus nephritis needs to be reappraised, as there is evidence from the United States that treatment based on histology in the absence of overt renal disease improves prognosis.

The role of renal transplantation and the effects that both this and end-stage renal failure have on extrarenal disease activity merit further study, as this may provide fascinating insights into the pathogenesis of renal and non-renal SLE.

\section{Acknowledgement}

We gratefully acknowledge the help given by Dr Guy Neild in the preparation of this manuscript. The meeting was sponsored by the generous support of Ciba-Geigy.

4. Bennett, W.M., Bardana, E.J., Houghton, D.C., Pirofsky, B. \& Striker, G.D. Silent renal involvement in systemic lupus erythematosus Int Arch Allergy Appl Immunol 1977, 55: 420-428.

5. Cavallo, T., Cameron, W.R. \& Lapenas, D. Immunopathology of early and clinically silent lupus nephritis Am J Pathol 1977, 87: 1-15.

6. Fries, J.F., Porta, J. \& Liang, M.H. Marginal benefit of renal biopsy in systemic lupus erythematosus. Arch Intern Med 1978, 138: 1386-1389. 
7. Whiting-O'Keefe, Q., Henke, J.E., Sheard, M.A., Hopper, J. JR, Biava, C.G. \& Epstein, W.V. The information content from renal biopsy in systemic lupus erythematosus. Stepwise linear regression analysis. Ann Intern Med 1982, 96: 718-723.

8. Whiting-O'Keefe, Q., Riccardi, P.J., Henke, J.E., Shearn, M.A., Hopper, J. JR \& Epstein, W.V. Recognition of information in renal biopsies of patients with lupus nephritis. Ann Intern Med 1982, 1: 723-727.

9. Dillard, M.G., Tillman, R.L. \& Sampson, C.C. Lupus nephritis. Correlations between clinical course and presence of electron dense deposits. Lab Invest 1975, 32: 261.

10. Tateno, S., Kobayashi, Y., Shigematsu, H. \& Hiki, Y. Study of lupus nephritis: its classification and the significance of subendothelial deposits. $Q J \mathrm{Med}$ New Series L II 1983, 207: 311-331.

11. Rosenbaum, J., Pottinger, B.E., Woo, P. et al. Measurement and characterization of circulating anti-endothelial cell IgG in connective tissue diseases. Clin Exp Immunol 1988, 72: 450-456.

12. Austin, H.A. III, Klippel, J.H., Balow, J.E. et al. Therapy of lupus nephritis. Controlled trial of prednisolone and cytotoxic drugs. $N$ Engl J Med 1986, 314: 614-619.

13. Balow, J.E., Austin, H.A. III, Muenz, L.R. et al. Effect of treatment on evolution of renal abnormalities in lupus nephritis. N Engl J Med 1984, 311: 491-495.

14. Carette, S., Klippel, J.H., Decker, J.L. et al. Controlled studies of oral immunosuppressive drugs in lupus nephritis. A long term follow-up. Ann Int Med 1983, 99: 1-8.

15. Kimberly, R.P. Renal prostaglandins in systemic lupus erythematosus. Lancet 1978, ii: 553.

16. Ziff, M. \& Helderman, J.H. Dialysis and transplantation in end-stage lupus nephritis (Editorial). $N$ Engl $J$ Med 1983, 308: 218-219.
17. Cameron, J.S., Lessof, M.H., Ogg, C.S., Williams, B⿳亠口冋. \& Williams, D.G. Disease activity in the nephritis of systemic lupus erythematosus in relation to serum complement concentrations, DNA binding and precipitafing anti-DNA antibody. Clin Exp Immunol 1976, 25: 418-427.

18. Pillemer, S.R., Austin, H.A. III, Tsokos, G.C. \& Balgुw, J.E. Lupus nephritis: association between serology and renal biopsy measures. J Rheumatol 1988, 15: 284-288.

19. Senaldi, G., Makinder, V.A., Vergani, D. \& Isenbefg, D.A. Activation of the fourth component of complemgnt (C4) reflects disease activity in systemic lupus erythematosus. Ann Rheum Dis 1988, 47: 913-917. हs

20. Stamenkovic, I., Faure, H., Donath, A., Assi苚copoulos, A. \& Chatelanat, F. Renal biopsy in systemic lupus erythematosus irrespective of clinical findings. Long term follow-up. Clin Nephrol 1986, 26: 109- Iㄷㅇㄱ.

21. Balow, J.E. Therapeutic trials in lupus nephritis: P blems related to renal histology, monitoring of theropy and measures of outcome. Nephron 1981, 27: 171-196.

22. Cheigh, 'J.S., Stenzel, K.H., Rubin, A.L., Chami, J; \& Sullivan, J.F. Systemic lupus erythematosus in patiefists with chronic renal failure. Am J Med 1983, 75: 602-606.

23. Coplon, N.S., Diskin, C.J., Peterson, J. \& Swenson, R The long term clinical course of systemic lupus erythematosus in end stage renal disease. $N$ Engl $\mathrm{J}$ Med 1983, 308: 186- 190.

24. Austin, H.A. III, Muenz, L.R., Joyce, K.M. et Prognostic factors in lupus nephritis: contribution of renal histologic data. Am J Med 1983, 75: 382-321 $\overrightarrow{0}$

25. Correia, P., Cameron, J.S., Lian, J.E. et al. Wh \&o patients with lupus nephritis die? $\mathrm{Br}$ Med $J$ 1985 200: $126-131$.

26. Leung, A.C.T. \& Boulton-Jones, M. Why do pattem with lupus nephritis die? (Correspondence). $\mathrm{Br} \mathrm{Me} \overline{\mathrm{C} J}$ 1985, 290: 937. 